\title{
Terapia ocupacional en el Perú: una historia de retos y desafíos
}

\author{
Occupational therapy in Peru: a history of challenges and challenges \\ Jaime Enrique Flores Rios ${ }^{1}$, Carlos Manuel Escobar Galindo ${ }^{1}$, Milagros de Jesús Céspedes Chauca ${ }^{1}$, Sarina \\ Francisca Ramos Zuñiga ${ }^{1}$, Alicia Encarnación García Palacios ${ }^{1}$
}

\section{RESUMEN}

La historia de la terapia ocupacional en el mundo se remonta a diferentes acontecimientos que marcaron su desarrollo, sin embargo, en el Perú no se cuentan con antecedentes claros que delimiten su evolución y la hagan visible a la comunidad peruana. Objetivos: Describir la historia, retos y desafíos de la terapia ocupacional en el Perú. Material y métodos: El estudio tuvo un diseño narrativo histórico -social centrándose en describir los acontecimientos sin establecer relaciones causales ni generalización. Las técnicas para recolectar información incluyeron, entrevistas, revisión de documentos y conclusiones de congresos. Resultados: La terapia ocupacional ha tenido grandes variaciones a lo largo de la historia que la consolidaron finalmente como una carrera profesional de la salud humanista y de servicio. Conclusiones: La terapia ocupacional en el Perú tiene más de 50 años como carrera profesional y actualmente tiene grandes retos y desafíos en la mejora de la salud y bienestar de la población con justicia y participación ocupacional.

PALABRAS CLAVE: Terapia ocupacional, Historia.

\section{SUMMARY}

The history of occupational therapy in the world dates back to different events that marked its development. However, there is no clear background to delimit its evolution in Peru to make it visible to the Peruvian community. Objective: To describe the history and challenges of occupational therapy in Perú. Material and methods: The study had a social-historical narrative design focusing on describing events without establishing causal relationships or generalization. The data collection techniques included interviews, document review and congress conclusions. Results: Occupational therapy has had major events throughout history that consolidated it as humanistic healthcare professional. Conclusions: Occupational therapy in Peru has been a professional career for more than 50 years and currently faces great challenges in improving the health and welfare of the population with justice and occupational participation.

KEYWORDS: Occupational therapy, History.

1 Escuela profesional de Tecnología Médica, Facultad de Medicina. Alberto Hurtado, Universidad Peruana Cayetano Heredia. Lima, Perú 


\section{INTRODUCCIÓN}

La terapia ocupacional en el mundo tiene una historia influenciada por diversas profesiones, eventos mundiales, personalidades y movimientos sociales que le han conferido su identidad actual como profesión(1). Es a partir de la historia, que la terapia ocupacional ha podido evolucionar y aprender de los diversos contextos vividos para llegar a ser una ciencia que va más allá de los sistemas de salud, tomando ahora incluso un alcance social y de desarrollo humano. Durante los diversos contextos históricos, la terapia ocupacional ha adquirido un contexto especial y particular que se ha adaptado en función a los requerimientos ocupacionales de cada nación, tomando como base un enfoque cultural y antropológico. Uno de los principales hitos en la historia mundial que marcaron un antes y después en la terapia ocupacional, fueron las ideas del tratamiento moral, en donde por primera vez se trataba con dignidad a los pacientes psiquiátricos y con disfunciones físicas (2) . Estas ideas evolucionaron y permitieron desarrollar nuevos enfoques terapéuticos que dieron base a distintos profesionales de diversas áreas y que posibilitaron implementar miradas holísticas de la ocupación, y que hoy en día son utilizados como base de la intervención en la terapia ocupacional $(3,4)$. Estos cambios de paradigma en la actuación terapéutica sumado al establecimiento de modelos sociales de rehabilitación médica y los nuevos modelos con base en comunidad participativa con justicia social, han influenciado en el establecimiento de perfiles profesionales en terapia ocupacional.

A pesar de que la historia de la terapia ocupacional a nivel mundial está claramente definida e identificada a través de los diferentes hechos y personaje históricos que contribuyeron a su desarrollo, en el Perú aún no existen cimientos claros sobre su desarrollo y evolución. Esta falta de información limita de alguna manera el contexto del quehacer actual al no tener clara los cimientos del crecimiento de la carrera. Actualmente, la carrera está creciendo principalmente sobre paradigmas específicos basados en contextos internacionales y no tomando en cuenta el propio contexto cultural y del entorno en el que se desenvuelven las personas en el Perú. Por otro lado, las necesidades de terapia ocupacional en la población siguen creciendo; sin embargo, la demanda es más alta que la oferta de profesionales capacitados en esta profesión (5).
Además, la falta de reconocimiento en los diversos momentos de la historia, así como los personajes que influyeron en el Perú, la vuelve invisible ante la comunidad restringiendo el desarrollo de la Terapia ocupacional en el país.

Por estas razones, el presente artículo tuvo como objetivo describir la historia de la terapia ocupacional en el Perú, retos y desafíos desde una perspectiva narrativa de los hechos y acontecimientos.

\section{MATERIAL Y MÉTODOS}

El estudio tuvo un corte principalmente reflexivo y narrativo histórico-social centrándose principalmente en describir los acontecimientos externos sin establecer relaciones causales ni generalización. Este análisis constituye un espacio importante de investigación debido a que permite establecer factores que expliquen su desarrollo y legitimización en la sociedad (6).

Para el desarrollo de la narrativa se desarrollaron entrevistas con tres terapeutas ocupacionales reconocidos por su trayectoria profesional en el Perú utilizando una técnica propositiva. También se revisaron documentos existentes en diferentes instituciones y se recopilaron conclusiones de diversos eventos científicos de terapia ocupacional durante los últimos cinco años.

\section{RESULTADOS}

\section{Inicios de la Terapia Ocupacional en el Perú}

La historia de la Terapia Ocupacional en el Perú está íntimamente relacionada a la emergencia de la profesión en otros países de Latinoamérica como México y Argentina a mediados de los años sesenta. Sin embargo, encontramos sus más antiguos orígenes en un artículo escrito por Hermilio Valdizán en 1937, donde se hablaba del tratamiento de la esquizofrenia, mostrando interés en implementar actividades de terapia ocupacional en el hospital psiquiátrico Victor Larco Herrera. Aunque no se cuenta con evidencia sustentable, se han registrado antecedentes de enseñanza de artes y oficios para personas con enfermedad mental. Es en el año 1962 que, por una beca auspiciada por la embajada de los Estados Unidos, dos maestras peruanas Vilma Cavero de Castro y Clemencia Vallejos, son seleccionadas para estudiar Terapia Ocupacional en el Instituto Mexicano de Rehabilitación bajo la dirección del 
Doctor Tohen Zamudio, ícono en el desarrollo de la Rehabilitación en Latinoamérica. El 14 de julio de 1962, en el gobierno del presidente Dr. Manuel Prado Ugarteche, se inaugura en el distrito de San Miguel el Instituto Nacional de Rehabilitación (INR) asumiendo como primera directora la Dra. Adriana Rebaza Flores. Es al año siguiente, que la terapeuta ocupacional Vilma Cavero de Castro se incorpora al Instituto y se apertura de manera formal el primer servicio de Terapia Ocupacional en el Perú. Posteriormente, en 1969, atendiendo a niños y adultos con discapacidades físicas. En 1969 se incorpora al Instituto de Rehabilitación la terapeuta ocupacional Luz María Camino quien se formó en Argentina. Es en 1969 que se apertura el primer programa académico de Terapia Ocupacional dentro de la Escuela Académico Profesional de Tecnología Médica por recomendación de la doctora Adriana Rebaza Flores en la Universidad Nacional Mayor de San Marcos (UNMSM), formándose las dos primeras promociones de terapeutas ocupacionales en el Perú. Posteriormente, en 1971 se apertura en la Escuela de Rehabilitación del Instituto Peruano de Seguridad Social la carrera de terapia ocupacional, siendo estudiada por una sola promoción y ocupando las plazas de los hospitales del seguro social, tales como el Hospital Obrero (Actualmente Hospital Nacional Guillermo Almenara Irigoyen) y el Hospital del empleado (actualmente Hospital Edgardo Rebagliati Martins). Esta escuela no dependía de ninguna universidad por ende se consideraba de nivel técnico. Las primeras promociones de terapeutas ocupacionales formados en la UNMSM se insertan laboralmente en diversos hospitales y centros nacionales de rehabilitación física y mental. Las primeras áreas de especialización que se dieron en la terapia ocupacional en el país fueron las de disfunciones físicas en adultos, niños y salud mental. Terapeutas ocupacionales como Enrique Sarmiento, Martha Veliz, Clara Sertzen, Bilma Avelino, Matilde Medina, Clelia Tapia, María Elena Benavidez, Irene Alakawa, Gladys Evangelista, Hilda Ayme y Nelly Salas, aperturaron diversos servicios de terapia ocupacional en hospitales generales y castrenses brindando atención a adultos y niños con discapacidad física y mental. En 1972 la coyuntura política social del país dada por la dictadura militar del General Velasco Alvarado, generó que algunos programas de las universidades públicas se cierren, entre ellos la carrera de Terapia Ocupacional. Posteriormente, en 1975 la terapeuta Alice Mendes, representante de la Federación Mundial de Terapeutas Ocupacionales, recomienda la formación de una asociación profesional que represente a los terapeutas ocupacionales en el
Perú, es ahí donde se forma la primera Asociación de Terapeutas ocupacionales en el Instituto Nacional de Rehabilitación, Instituto Nacional de Rehabilitación. La Asociación estuvo conformada por los Licenciados Luz María Camino, Enrique Sarmiento y Vilma Cavero de Castro como Presidenta, sin embargo por diversos motivos no pudo ser registrada formalmente. Es así que, en 1978, se reabre la carrera de Terapia Ocupacional en la UNMSM con el grado académico de bachiller en Tecnología Médica, y licenciatura en Tecnología Médica en el área de Terapia Ocupacional con una duración académica de 5 años; consolidando así su alcance profesional. Finalmente, el 25 de setiembre de 1987 se constituye la primera asociación de Terapeutas Ocupacionales del Perú con el nombre de Asociación Peruana de Terapeutas Ocupacionales (APTO), siendo su primera presidente la Lic. Matilde Medina. El objetivo fue representar de forma gremial a los Terapeutas Ocupacionales del Perú y velar por los estándares de formación y ejercicio profesional. En el año 1999 la Universidad Peruana Cayetano Heredia (UPCH) apertura el segundo programa académico siendo la primera universidad privada en el país en formar terapeutas ocupacionales. En el año 2000, Perú logra ser parte de la Confederación Latinoamericana de Terapia Ocupacional CLATO que se fundó en 1998 en el marco del III Congreso Latinoamericano de Terapia Ocupacional en Caracas Venezuela. Es en el año 2001 donde se dictan las primeras jornadas nacionales de Terapia Ocupacional y se estípula que el 25 de septiembre se celebre el Día del Terapeuta Ocupacional; día para reconocer la labor profesional y reasumir el compromiso de crecimiento y difusión de la profesión a nivel nacional. Entre los años 2001 y 2003, la APTO, organizó y auspició cursos de formación de distintas especializaciones tales como en integración sensorial y el modelo de la ocupación humana, dando empuje y fortaleza a la profesión y brindando la oportunidad a muchos colegas que se formaran en Marcos de Referencia relevantes para la profesión. Esta asociación se mantuvo vigente hasta el año 2005; sin embargo, debido a dificultades con las sucesiones de las juntas directivas, la última junta directiva presidida por el Lic. Jaime Flores en asamblea general deciden formar una nueva asociación gremial que represente a los Terapeutas Ocupacionales en el Perú, fundándose la Asociación Profesional de Terapeutas Ocupacionales del Perú (APTOP) con fecha 15 de septiembre del 2006 , como consta en los registros públicos, siendo su primer presidente el Lic. Jaime Flores. La APTOP tuvo como uno de sus principales objetivos pertenecer a la Federación Mundial de Terapeutas Ocupacionales, hito histórico 
que se logró ese mismo año en calidad de miembro asociado.

En el año 2009 se celebra en Lima el Primer Congreso Peruano y VIII Congreso Latinoamericano de Terapeutas Ocupacionales que contó con la presencia de la presidenta de la Federación Mundial de Terapeutas Ocupacionales la Dra. OT Sharon Brintnell, recayendo la presidencia de la Confederación Latinoamericana de Terapeutas Ocupacionales (CLATO) al Perú, asumiendo el cargo la Lic. Clara Sertzen y estando como presidenta de la APTOP la Lic. Marlene Machuca. En la actualidad la Terapia Ocupacional es una profesión reconocida principalmente en las áreas de la rehabilitación, tecnología asistiva, educación básica regular y especial, salud mental, ergonomía y áreas emergentes como el deporte.

\section{Inicios de la Terapia ocupacional en la Universidad Nacional Mayor de San Marcos}

La formación profesional del Terapeuta Ocupacional en el Perú está vinculada a la apertura de la Escuela Académico Profesional de Tecnología Médica la cual se crea por acuerdo del Consejo de la Facultad de Medicina de la UNMSM siendo Decano de la Facultad el Dr. Jorge Campos Rey De Castro el 1 de diciembre de 1966. Ese mismo año, se aprobaron las normas generales de su organización como dependiente de la Facultad de Medicina de la UNMSM, siendo considerada como una carrera universitaria corta, de tres años de duración, con un ciclo básico de dos semestres un semestre de especialización de dos años y un semestre de práctica profesional. Las primeras áreas de formación fueron Las primeras áreas de formación fueron Radiología, Histología y Citodiagnóstico y Laboratorio Clínico. siendo el primer director de escuela el Dr. Manuel Torres Portugal. El gobierno militar de ese entonces dirigido por el General Juan Velasco Alvarado, promulga en 1969 la Ley Orgánica 17437 eliminando así el sistema facultativo de la Universidad Peruana e instaurando como base de la organización universitaria el "Sistema Departamentalista" y de Programas Académicos. En mayo de 1969 se lleva a cabo la adecuación académica de la Universidad, implementándose los diferentes Departamentos Académicos con la ubicación de las carreras profesionales en Programas Académicos y el "ciclo básico". La nueva reestructuración coloca en tela de juicio que Tecnología Médica se constituya en Escuela Académico Profesional, al ser considerada "carrera de mando medio" y se propone transferirla a la Escuela de Estudios Especiales, que funcionaba como un Centro de Extensión Universitaria. Esta situación conlleva a una serie de reclamos y luchas estudiantiles lográndose mantener el título de Tecnólogo Médico y viéndose la necesidad de implementar una nueva especialidad denominada Terapia Ocupacional. A mediados de 1972, egresa la primera promoción de terapeutas ocupacionales y a finales del mismo egresa la segunda promoción; sin embargo, la escuela fue declarada en extinción y permaneció cerrada por 2 años, sin nuevos ingresantes. Es para 1973 que se reestructura la Escuela de Tecnología Médica y se logra la reapertura de la carrera con una duración de formación universitaria de cuatro años, sin embargo, debido a la falta de estudiantes de terapia ocupacional el programa no fue abierto sino hasta 1978.

En los años 1980 - 1981, sucede una segunda gran reestructuración curricular, la UNMSM aprueba el proyecto de restructuración académica-administrativa del programa de Tecnología Médica mediante Resolución Rectoral $\mathrm{N}^{\circ}$ 62550, convirtiéndose en carrera con una duración de 10 semestres académicos (5 años), incluido un año de internado, otorgando el grado de Bachiller y el título profesional de Licenciado en Tecnología Médica con mención al área de Terapia Ocupacional. Este currículo con muy pocas modificaciones en su plan de estudios se mantuvo hasta el año de 1996. Es a partir del año 1992 que se aprueba el ingreso directo al programa de Terapia Ocupacional, ingresando promociones continuas hasta la actualidad. En 1996 se realiza la tercera reforma curricular que incorporó cursos más específicos y especializados Terapia ocupacional en geriatría y medicina interna, ergonomía, análisis del ambiente y barreras arquitectónicas. En enero del 2001, se realiza un seminario de evaluación curricular que introduce modificaciones en el plan de estudios aprobado en 1996 y se establece la cuarta reforma curricular.

En el año 2005 se aprobó la Ley No 28456, "Ley del Trabajo del Profesional Tecnólogo Médico" y a continuación en el 2009, se inicia un nuevo proceso de evaluación curricular que culmina con RR 03803R-11 del 19 de julio del 2011 cuya finalidad fue dar mayor independencia a las carreras profesionales de Tecnología Médica. Finalmente, con RR 03883-R12 del 19 de julio del 2012, aprueban lo que seria el sexto plan curricular de la EAP de Tecnología Médica tomando en cuenta los estándares establecidos en el Modelo de Calidad para la Acreditación de la carrera profesional de Tecnología Médica. Posteriormente en el 2018 con RR 07694-2018 aprueban el séptimo plan curricular que está vigente hasta la actualidad. 
Este último plan se desarrollo en base a la reciente Ley Universitaria 30220 incluyendo ahora estudios generales a todas las carreras alineándose al modelo educativo de la Universidad de San Marcos y la Superintendencia Nacional de Educación Superior (SUNEDU).

\section{Inicios de la Terapia ocupacional en la Universidad Peruana Cayetano Heredia}

La UPCH, como entidad universitaria comprometida y atenta a las necesidades y formación de recursos humanos del país, creó la Escuela de
Tecnología Médica el 30 de junio de 1997 por acuerdo de Consejo universitario como parte de la Facultad de Medicina (RR N ${ }^{\circ}$ 426-97-UPCH-R del Documento D-FMAH-467-97 de fecha 21 de marzo de 1997. Creación de la Escuela). Posteriormente, en el año 2003 se crea el Departamento de Tecnología Médica (RR No009-2002-UPCH-AU del 20 de diciembre del 2002), las raíces de las mismas se originan gracias al Dr. Germán Benito Aragón gestor del proyecto de creación de la escuela de Tecnología Médica, quien convocó a un grupo de profesionales en los meses de setiembre - octubre del 1996, entre las que destacan la Dra. Lucrecia Tompson (Médico Rehabilitador),
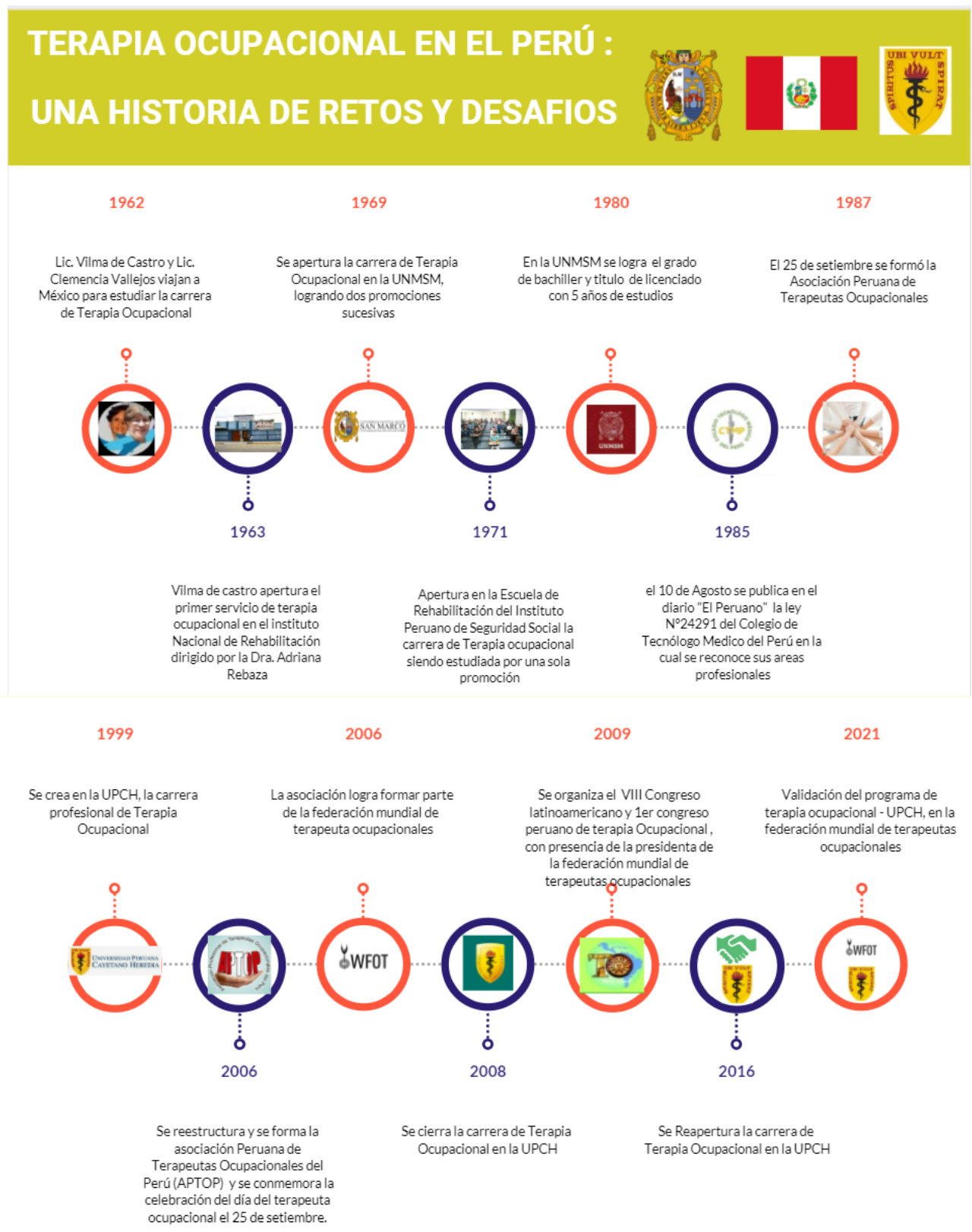

Figura 1. Línea del Tiempo de diversos hechos acontecidos en la historia de la Terapia Ocupacional peruana 
Dra. María García ( Médico Radiólogo) y el Dr. Jorge Atuncar (Médico Internista). Desde su fundación la escuela se trazó la meta de formar profesionales altamente calificados con una sólida formación en conocimientos y valores éticos, humanísticos de vocación de servicio contribuyendo a mejorar la calidad de vida de la población desde la investigación. Inicialmente en el año 1997 se formaban tecnólogos médicos en Laboratorio Clínico, posteriormente en el año de 1998 comenzaron sus actividades académicas las especialidades de Terapia Física y Rehabilitación, Urgencias Médicas y Radiología. En el año de 1999 se incorporaron tres especialidades más: Terapia de Audición Voz y Lenguaje, Biomecánica y Terapia Ocupacional por RR N ${ }^{\circ}$ 079-99-UPCH-CU del 26 de febrero del 1999.

La Escuela de Tecnología Médica de la Facultad de Medicina Alberto Hurtado, fue la primera entidad educativa privada en formar licenciados Tecnólogos Médicos en la especialidad de Terapia Ocupacional. Durante casi 10 años de actividad académica se formaron 29 Terapeutas Ocupacionales; sin embargo, en el año 2008 la especialidad de Terapia Ocupacional tuvo que cerrar sus actividades. Ante el creciente aumento de esperanza de vida en la población, incremento de enfermedades no transmisibles, enfermedades emergentes, del desarrollo humano, incremento de violencia en la sociedad, problemas de salud mental, así como accidentes y enfermedades relacionadas a la salud ocupacional se reapertura la carrera profesional por Resolución Rectoral SEGENUPCH-2016-CU 0059 el 27 de enero del 2016 gracias a la gestión del Dr. Jesús Tamariz como Director de la Escuela profesional de Tecnología Médica . Así mismo, la carrera profesional de Terapia Ocupacional inició su segunda etapa trazándose objetivos claros para empoderarla en la sociedad. Estos objetivos consistieron en incentivar la formación de nuevos conocimientos a través de la investigación, implementar programas de posgrado y formación continua y lograr la acreditación internacional de la carrera de Terapia Ocupacional. Como uno de los principales hitos en el año 2022 la carrera de Terapia ocupacional en la Universidad Peruana Cayetano Heredia será reconocida como Carrera Acreditada por la Federación Mundial de Terapia Ocupacional, siendo el primer programa académico en el Perú con esta distinción.

A modo de resumen general, la figura 1 describe los principales hitos a lo largo de la historia de la Terapia ocupacional peruana.

\section{DISCUSIÓN}

La Terapia Ocupacional es una profesión de la salud que busca mejorar la calidad de vida de las personas optimizando su desempeño ocupacional a través de la actividad con propósito y la ocupación, para garantizar una participación justa y equitativa en la sociedad. $\mathrm{Su}$ evolución a lo largo de los años demuestra el compromiso de los diferentes profesionales de la salud, así como de terapeutas ocupacionales quienes desarrollaron labores en favor de la participación ocupacional e integración sociolaboral de las personas y que ahora con justicia son reconocidos como parte de la historia de la terapia ocupacional en el Perú.

La historia señala que la Terapia ocupacional en el Perú comenzó con la búsqueda de la mejora del trato a personas con problemas de salud mental similar a lo ocurrido en la historia mundial con el tratamiento moral (2). Sin embargo, hoy en día, es el área donde menos terapeutas laboran y en donde menos cursos formativos o especializados se desarrollan (5), a pesar que en esta área la demanda actualmente se ha acrecentado enormemente con la formación de centros de salud mental comunitarios en el marco de la Ley General de Salud Mental publicada en el año 2019 y su reglamento $(7,8)$. Este y otros acontecimientos nos llevan a una reflexión hacia donde debemos ir como profesión y que debemos rescatar como base para alimentar los paradigmas que guían nuestro quehacer profesional.

Por otro lado, la historia nos enseña también que han pasado 50 años y sólo dos universidades vienen formando terapeutas ocupacionales a nivel nacional, lo que termina por limitar el acceso de intervenciones de terapia ocupacional en la población peruana, observándose poca presencia de terapeutas ocupacionales en el interior del país y en diferentes ámbitos de aplicación. El escaso número de terapeutas ocupacionales restringe la posibilidad de lograr inclusión e integración social y laboral en la sociedad peruana. Los futuros desafíos deben estar enfocados en aperturar nuevos programas en centros de estudios de prestigio y desarrollar especializaciones de post grado que fomenten la investigación y nos permitan consolidar nuestra actuación profesional en todo el país.

Finalmente, es gracias a la recopilación histórica que se puede reflexionar sobre los retos actuales que afronta la terapia ocupacional, permitiendo afianzar la identidad como terapeutas ocupacionales, 
y empoderando a los profesionales para confrontar los desafíos del mañana, colaborando así en el crecimiento y desarrollo de la profesión. Una profesión comprometida desde sus orígenes en la búsqueda de la equidad, de la justicia y de la participación ocupacional en la población respetando la diversidad y la riqueza cultural del Perú.

Agradecimientos: Un agradecimiento especial a lo(a) s licenciado(a)s en Terapia Ocupacional Vilma Cavero Vda de Castro; Clara Sertzen Fernández; Enrique Sarmiento Hurtado; Ada Ramos y Luz María Camino Ivanisevich ; quienes contribuyeron a través de sus testimonios y experiencias al desarrollo del estudio; así mismo a la Dra. Lucrecia Thompson y al Dr. Jesús Tamariz , por su gentil colaboración en el desarrollo de la historia en la UPCH . De igual manera, un agradecimiento y un homenaje especial a quien en vida fue la Maestra Paula Martha Veliz Terry, terapeuta ocupacional quien dedicó su vida al desarrollo de la Terapia Ocupacional en el Perú desde sus inicios en la Universidad Nacional Mayor de San Marcos.

\section{Correspondencia}

Carlos Manuel Escobar Galindo

Correo electrónico:manoergo@gmail.com

Conflicto de interes: Los autores declaran no tener conflicto de intereses.

\section{REFERENCIAS BIBLIOGRÁFICAS}

1. Kielhofner G. Fundamentos conceptuales de la terapia ocupacional. Buenos Aires: Ed. Médica Panamericana; 2006.

2. Christiansen $\mathrm{CH}$, Haertl K. A contextual history of occupational therapy. Willard \& Spackman's occupational therapy. 2014;1: 9-34.

3. Turpin MJ, Iwama MK. Using Occupational Therapy Models in Practice E-Book: A Fieldguide. New York: Elsevier Health Sciences; 2011.

4. Kielhofner G. Terapia ocupacional: modelo de ocupación humana: teoría y aplicación. Buenos Aires: Ed. Médica Panamericana. 2014.

5. Escobar C, Trinidad J, Céspedes M, Reyes M, Cárdenas A. E. Especialización en Terapia Ocupacional: una oportunidad para el Perú. Rev Her Rehab. 2020;3(1).

6. Alatorre E. La investigación histórica como estrategia para la transformación de la enfermería. Enfermería universitaria. 2010;7(5):06-12.

7. Congreso de la República. Ley de Salud Mental 30947. Lima: Diario El Peruano; 30 de abril del 2019. (Citado el 20 de setiembre del 2020) Disponible en: https://busquedas.elperuano.pe/normaslegales/leyde-salud-mental-ley-n-30947-1772004-1/

8. Ministerio de Salud. Reglamento de Ley de Salud mental $\mathrm{N}^{\circ}$ 30947. Lima: Ministerio de Salud; 5 de marzo del 2020.

Recibido: 12/02/2021

Aceptado: $15 / 04 / 2021$ 